\title{
PROCEDURAL EMBODIMENT AND MAGIC IN LINEAR EQUATIONS
}

\author{
Rosana Nogueira de Lima ${ }^{1}$ \\ David Tall \\ PUC/SP - Brasil \\ University of Warwick - UK
}

How do students think about algebra? Here we consider a theoretical framework which builds from natural human functioning in terms of embodiment-perceiving the world, acting on it and reflecting on the effect of the actions-to shift to the use of symbolism to solve linear equations. In the main, the students involved in this study do not encapsulate algebraic expressions from process to object, they do not solve 'evaluation equations' such as $3 x+2=8$ by 'undoing' the operations on the left, they do not find such equations easier to solve than $3 x-1=3+x$, and they do not use general principles of 'do the same thing to both sides'. Instead they build their own ways of working based on the embodied actions they perform on the symbols, mentally picking them up and moving them around, with the added 'magic' of rules such as 'change sides, change signs'. We consider the need for a theoretical framework that includes both embodiment and process-object encapsulation of symbolism and the need for communication of theoretical insights to address the practical problems of teachers and students.

\section{INTRODUCTION}

In this paper we review the responses of three classes learning algebra in Brazil in a project with six teachers who agreed to collect data and reflect on the result of their regular teaching of mathematics in the Brazilian curriculum. Students were taught using the general principle of 'do the same thing to both sides' of the equation to retain the equality. The data collected does not fit the 'didactic cut' in which students are more successful dealing with linear equations with a number on the right hand side (which can be 'undone' using arithmetic alone) than with equations with expressions on both sides. Instead many students respond to the general principle of 'doing the same thing to both sides' in specific terms in which the +1 in $5 x+1=3 x+5$ is shifted over the other side with a 'change in sign' and the 3 in $3 x=6$ is 'moved over the other side and 'put underneath".

This shifting of symbols is seen as an application of human embodiment, picking up things and moving them around. It works fine for simplifying expressions such as $4 a+3 b+2 a$ where the term $2 a$ can be shifted next to the $4 a$ and combined to give $6 a+3 b$, but fails for equations where shifting terms to the other side requires additional actions (change signs, put it underneath). If this symbol-shifting is not set

\footnotetext{
${ }^{1}$ The first author was supported by the CAPES Foundation, Ministry of Education of Brazil.
} 
in a meaningful context, the manipulation may be seen as moving symbols with a kind of additional 'magic' to get the correct solution.

Given the implicit use of embodiment in shifting symbols around, we set our analysis in a theoretical framework that sees mathematical thinking begin in conceptual embodiment based on human perception, action and reflection and broaden to include proceptual symbolism, in which dynamic actions, such as counting, are symbolised so that the symbols may be used dually as process and concept (procept). This is part of a longer-term theory of cognitive development where the properties and inferences developed in conceptual embodiment and proceptual symbolism are later reformulated in advanced mathematical thinking in terms of axiomatic theories given by concept definitions and mathematical proof (Tall 2004a,b).

In section 2 we describe the theoretical framework and relate it to the literature. Sections 3 and 4 present the algebraic tasks set and analysis of the data. Section 5 discusses the implications for the development of a broader theory encompassing both embodiment and symbolism, and its consequences for the practice of teaching algebra in particular and mathematics in general.

\section{THEORETICAL FRAMEWORK}

The encounter with linear equations is part of a much broader development of mathematical thinking that occurs in the life of the individual from new-born baby to mature adult. As such, it sits within a context that should take into account what went before and what may happen after, in particular, the way in which the human mind builds on human perception and action to lead to symbolic thinking in mathematics.

Learning occurs by making new connections in the brain. This happens when a sequence of actions is repeated until it becomes automatic. It relegates the routine to subconscious activity, leaving conscious thought to think about important issues in any given context. More powerful connections are made using words and symbols to focus on relevant aspects of experience that become 'thinkable concepts' used in 'thought experiments' to imagine new possibilities and to grow in sophistication. This process of making links leads to a compression of knowledge from complicated phenomena to rich concepts with useable properties and coherent links to other ideas. Compression of knowledge is at the heart of human thought in general and mathematical thinking in particular (Thurston, 1990).

Piaget theorized that there are two qualitatively different ways of operating on the external world, which he described as empirical and pseudo-empirical abstraction: one focusing on objects and their properties, the other focusing on the actions on the objects. These lead to two distinct modes of compression to construct thinkable concepts: one building a mental world of conceptual embodiment of the objects we perceive and think about, the other in which reflection on actions leads to their encapsulation as mental objects. 
The encapsulation of actions, via step-by-step procedures, then as input-output processes, and as mental objects in their own right is well-represented in the literature (eg. Dubinsky, 1991; Sfard 1991). However, the actual shift from process to object seems to be a difficult one for many students to accomplish.

When symbols are first manipulated, the focus is naturally on the sequence of steps performed, and there is initially considerable mental effort involved in performing the steps to transform $(a-b)(a+b)$ into $a^{2}-b^{2}$. At this stage, the two expressions are likely to be seen as different things, with one thing being transformed into the other. However, if the focus changes to the effect of the expressions in terms of evaluation for numerical values of $a$ and $b$, then they are just different ways of expressing the same thing. This phenomenon, where symbols represent both a mental concept and also possibly different actions to attain the same effect is called a procept (Gray \& Tall, 1994). The idea of giving embodied meaning to procepts in terms of the effects of the actions was introduced in Watson (2002) and Watson, Spyrou \& Tall (2003). The shift from many actions to a single thinkable concept is an act of compression that is an essential tool in making profound sense of mathematics.

The term 'effect' has been used before in elaborating the nature of pseudo-empirical abstraction. For instance, Simon et al. (2004) have focused on the relationship between activity and effect where both the activity and its effect (as noticed by the learner) serve as a basis for a new conception. Our use of the term relates more specifically to the desired mathematical effect of a given procedure, which we would hope to convey to the learner. For example, the fractional symbols $2 / 4$ and $3 / 6$ represent possible different activities: divide something into 4 equal parts and select 2 , or divide it into 6 parts and take 3; these have the same effect in terms of quantity, but may have different effects in terms of the actions performed or the number of pieces produced. Traditionally this relationship between fractions is described in terms of equivalence, which proves to be a stumbling block for many children; we would suggest that focusing on the observation that two such actions have the same effect is a more immediate way to make sense of the essential idea.

The distinction between empirical and pseudo-empirical abstraction involves different ways of compressing knowledge structures into thinkable concepts. Over the long term, the modes of operation themselves become more sophisticated, suggesting two quite different mental worlds of mathematics:

- a conceptual embodied world of human perception and action (including pictures, mental images and the internal connections we make in our mind);

- a proceptual symbolic world of mathematical symbols that operate flexibly either as concepts to think about or as processes to make calculations and perform symbolic manipulations.

These ways of thinking operate in tandem throughout school mathematics. A third world of mathematical thinking grows out of the properties observed in school mathematics, but will not concern us here: 
- the axiomatic formal world of formal mathematics expressed as axiomatic systems, formal definitions and mathematical proof that is met in pure mathematics at university.

These three 'worlds' have many resonations with a range of other theories in cognitive development (Tall 2004a, 2004b). However, they are more than modes of operation as each world develops in sophistication as the learner matures, with embodiment following a van Hiele like development and symbolism developing through process-object encapsulation. The interplay between the worlds of embodiment and symbolism proves to be crucial. A most important aspect is how the symbolic development from process to object is mirrored in a shift in focus from the steps of an action to the effect of the action. This will feature strongly in the theoretical framework of this paper.

\subsection{The notion of met-before}

In our analysis, we see students building on experiences that they have met before. Experiences in counting affect our conceptions of number, experiences in arithmetic affect our conceptions of algebra, ways of operating that have been seen to work at one time are brought to mind in attempting to make sense of a new context. Following Tall (2004b), we define a 'met-before' to be a mental construct that an individual uses at a given time based on experiences they have met before.

A met-before is a specific part of the individual's concept image, however, by giving it a specific name, we intend that it can be used for students and teachers to discuss openly how their previous knowledge can affect their interpretation of a new context.

For instance, different kinds of meaning given to symbols in algebra arise from previous experiences such as those encountered in arithmetic and the underlying conceptual embodiments that are natural consequences of human functioning: picking up things and moving them, collecting together like things and combining them.

Met-befores can influence current learning in both positive and negative ways. If a child knows ' $2+2$ is 4' from real-world experiences, it is still an essential piece of knowledge throughout future learning. But if the child implicitly relates this to other experiences of adding whole numbers, such as sensing that 'addition always gives a bigger result', then this will act as a possible difficulty when adding two numbers where one or both may be negative. Met-befores can cause problems when they are used out of their domain of validity, in a manner that has been described as an obstacle by Brousseau (1997) in the context of mathematics education.

In looking at the longer-term development of conceptual growth, we note that not only do met-befores affect new learning, but new experiences may affect the way we conceive of old knowledge. For instance, Anderson (1997), showed clearly how experiences in arithmetic - such as the fraction bar in $\frac{12}{6}$ triggering the process of 
'dividing the number at the top by the number at the bottom' to give the correct answer 2-may be incorrectly applied in algebra, to operate on $\frac{a^{12}}{a^{6}}$ to give the erroneous result $a^{2}$. On the other hand, experiences in algebra, such as simplifying $5 x^{2} \times 4 x^{3}$ to give $20 x^{5}$ by 'multiplying the coefficients and adding the powers' can be misapplied in arithmetic to wrongly simplify $5^{2} \times 4^{3}$ as $20^{5}$. There is therefore a symmetry in the way that experiences met before can affect learning now, but also experiences met afterwards can affect memories of old learning. We use the term 'met-after' to denote an experience met at a later time that affects the memories of previous knowledge.

\subsection{Embodiment in arithmetic and algebra}

Our perception of the problems facing students beginning algebra is that, unlike arithmetic - where the addition of whole numbers corresponds to physical actions on objects - algebra works at a more general level and various embodiments that occur may prove to be more problematic. Actions on simple linear expressions - such as simplifying $3 a+4 b+2 a$ by collecting like terms to give $3 a+2 a+4 b$ then simplifying to $5 a+4 b$ - can be conceived in an embodied way by 'moving' the $2 a$ 'next' to the $3 a$ and combining them together to get $5 a$. In the mind's eye, this may be imagined as 'picking up' the ' $2 a$ ' and 'moving it over' the ' $4 b$ ' to get 'like terms' together. When imagining $a$ and $b$ as objects rather than variables that can take numerical values, this is sometimes conceived as a kind of 'fruit salad algebra' where $a$ is for apple and $b$ is banana. However, it does not take much variation to cause difficulties for students (and teachers) handling expressions. (See, for example, Tirosh, Even \& Robinson, 1998). For instance, faced with $3+2 x$, a student not knowing what $x$ is may recognise the part $3+2$ and add these together using the metbefore from arithmetic and leave the $x$ as it is to get $5 x$.

\subsection{Evaluation algebra in equations as operations}

The shift from operating on linear expressions to working with linear equations involves a further change in meaning. Kieran (1981) reported that 13 year-old students use the equals sign not to indicate equivalence, but as an operational sign that entices them to perform the operations before the sign and put the solution after it.

This view can be useful dealing with a simple linear equation like $3 x+2=8$ with an expression on the left to be calculated and a number on the right. It may be seen as an operation $3 x+2$ to be evaluated, multiplying a number by 3 , adding 2 to get 8 . It may be 'undone' (Hart et al., 1989) by taking the 2 off the 8 to get $3 x=6$ and so seeing that $x$ is 2 . In this way an equation of the form 'expression equals number' may be solved by simply using arithmetic operations.

More generally, algebraic expressions may be used in spreadsheets to evaluate a particular expression in a way that has become very important in the today's 
technology using spreadsheets. Tall and Thomas (2001) described three kinds of algebra: evaluation algebra, in which algebraic expressions are evaluated, manipulation algebra, in which algebraic expressions are manipulated to simplify expressions and solve equations, and axiomatic algebra, in which algebraic systems such as vector spaces or systems of linear equations are handled in the formal axiomatic world of concept definition and formal proof.

Within this framework, we will use the term 'evaluation equation' to describe an equation of the form $3 x+2=8$ where an expression is evaluated to give a number. However, there is a significant change in meaning between an evaluation equation, where the expression on the left can be seen as a process, and a more general equation where there are non-trivial expressions on both sides.

\subsection{More complicated equations: symbolic manipulation and embodiment}

Linear equations with the unknown on both sides, such as $3 x+2=8+x$, cannot be solved by 'undoing the arithmetic operations'. The increased level of difficulty in solving these equations was christened the 'didactic cut' by Filloy and Rojano (1989); they reasoned that it would be more difficult for students to find the required value because they now have to operate on the unknown and not just on numbers, as they may have done with evaluation equations. From a process-object viewpoint, if the expressions on each side are seen only as different procedures of evaluation, it is not easy to think of 'undoing' both operations simultaneously, instead it is easier to think of them as mental objects (or groups of mental objects) that can be manipulated mentally.

One way forward which retains an embodied meaning is to conceptualise an equation such as $3 x+2=8+x$ as a 'balance' with 3 'things' and 2 units on one side and 8 units and one 'thing' on the other. These 'things' might be containers with the same number of units inside them. Balance is retained by 'taking the same item from both sides', for instance, removing 2 units from each side to leave ' 3 things on one side' and ' 6 units and a thing on the other' $(3 x=6+x)$, then removing a thing from each side leaves 2 things balancing 6 units $(2 x=6)$, so $x$ is 3 . This method works equally well with evaluation equations such as $3 x+2=8$ so this embodiment seems, on the face of it, superior to the method of solution by 'undoing'.

However, it is not. The equation $2 x-3=5$ is an evaluation equation that can be solved by 'undoing', but it is less easy to see as a 'balance' because it is not possible to subtract the 3 from $2 x$ on the left-hand side if $x$ is not known. The 'balance' embodiment becomes more difficult to interpret meaningfully when subtraction or negative quantities are involved. One possibility is to represent taking off 3 on the left hand side by putting 3 on the right hand side. Another is to imagine helium balloons that lift up the balance and so act as negative numbers. Such problems even arise in equations that have all positive-looking quantities in them such as $2 x+3=2+x$, which seems at first sight to be amenable to a physical representation, yet reduces to $x+1=0$, which seems impossible. 
Vlassis (2002), using equations with the unknown on both sides, showed that the balance model was a helpful metaphor for almost all his students in giving meaning to the equals sign as an equality between the two sides of the equation. However, it failed to be meaningful for many students in more general situations involving subtraction and negative numbers. An embodiment can clearly be supportive in situations where it is directly applicable but may then cause an impediment when it no longer applies in a new context.

\subsection{Symbol shifting as an embodied action with a difference}

The wider range of students who focus naturally on the detail and practice the procedures face difficulties in shifting from arithmetic to algebra, compounded by the limitations of 'undoing' and of simple embodiments for equations. Without meaningful embodiment or compressed symbolic meaning, there is a natural tendency to build on what they already know. The embodied met-before of shifting symbols around in expressions fails when moving symbols over the equals sign. To shift the number 2 in the equation $3 x+2=8$ to be next to the number 8 requires not only 'changing sides', it also requires the mystical operation of 'change signs' to get $3 x=8-2$. and to be able to perform the subtraction operation to get $3 x=6$. To solve this equation by 'moving the 3 over' involves shifting it over the other side, but now it needs to be 'put underneath' to get

$$
x=\frac{6}{3} \text {. }
$$

In this way, the student who is building on the met-before of embodied operations that work with simple linear expressions may easily continue to perform mental actions on the symbols ('seeing' objects, 'picking them up', and 'moving them') while adding 'magic' ingredients such as 'change sides change signs' or 'move it over and put it underneath'.

Cortés and Pfaff (2000) found that the principles used by the 17-year old students to solve equations in their study were all based on the 'movement' of symbols from one side to the other of an equation, as if the symbols were physical entities that students 'pass' to the other side of the equation with additional changes of signs or a shift to place it below the number in the other side. Freitas (2002) found that procedures related to phrases such as 'change side, change sign' were usually meaningless to students and often resulted in mistakes. As procedures, they are likely to become more complicated as the mathematics gets more sophisticated, and the increasing complication will cause even greater problems at later stages.

The question therefore remains: how do we help students negotiate the shift from arithmetic to algebra with the subtle changes from embodiment to symbolism? In Brazil, where the data to be reported in this paper was collected, documents that guide the teaching strategies and curriculum design for students in compulsory school levels (Brasil, 1998) emphasise the importance of giving meaning to algebraic symbols and using them to model real-life situations. However, it is not clear what 
kind of meaning is intended to be emphasised. Our analysis suggests that this meaning comes from human interaction with the world: things 'make sense' because they work in an expected way and if we look at them from a different perspective we can relate it to what we already know. Meaning therefore depends on our human perception and action and how we build on this and on our earlier experiences. The shift from arithmetic to algebra requires significant changes in meaning.

\section{THE TASK}

As part of a broader project on the teaching and learning of mathematics, the first author worked with six Brazilian mathematics teachers in weekly sessions in which they discussed pedagogical and mathematical aspects of equations. The discussion involved the teachers' conceptions of equations, the problems they have in teaching the subject, the difficulties their students face when solving equations, and relevant research papers (e.g. Freitas, 2002 and Vlassis, 2002). In these sessions, the teachers agreed to help the researcher to collect data from their students in order to make a picture of what may be the students' conceptions of equations, what solving methods they use and what previous experiences interfere in their work with equations. Together, teachers and researcher designed several instruments to collect data, including conceptual maps, a written task including algebraic problems and interviews. In this paper, we analyse students' work on linear equations presented in the written task, with the support of students' comments in subsequent interviews.

Three linear equations that students were asked to solve were:

$$
\begin{aligned}
& 5 t-3=8, \text { which can be 'undone' by arithmetical reasoning, } \\
& 3 x-1=3+x, \text { which has the unknown on both sides of the equation, and } \\
& 2 m=4 m \text {, which was suggested by one of the teachers as causing great } \\
& \text { difficulty among his students. }
\end{aligned}
$$

A test lasting 50 minutes was given in a regular mathematics class supervised by the classroom teacher consisting of these questions and four quadratic equations. There were 68 students in three groups of 15-16 year-old high school students: 22 first year and 28 second year from a public school in Guarulhos/SP, and 18 second year students from a private school in São Paulo/SP.

\section{RESULTS AND ANALYSIS}

In general, students had as much difficulty with the equation $3 x-1=3+x$ as with the equation $5 t-3=8$, judging by the number of students that solved them correctly.

\begin{tabular}{|c|c|c|c|}
\hline Equation & $5 t-3=8$ & $3 x-1=3+x$ & $2 m=4 m$ \\
\hline Successful & 25 & 25 & 7 \\
\hline Blank & 16 & 11 & 27 \\
\hline Other solutions & 27 & 32 & 34 \\
\hline
\end{tabular}


Table 1: Students' solutions of the equations.

The successful solutions for the first two equations were all similar. From $5 t-3=8$, they write $5 t=8+3$, then $5 t=11$, and therefore $t=\frac{11}{5}$. For the equation $3 x-1=x+3$, the solving steps were of the form $3 x-x=3+1$, that turns into $2 x=4$ and then $x=2$. Just sixteen students out of 68 solved both these equations correctly.

The theory of the didactic cut (Filloy \& Rojano, 1989) would suggest that the first question would have more successful responses than the second, but this did not happen. The probable reason for this was that the students attempted to stick closely to the method of solving linear equations by performing the same operation on both sides to preserve the equality. From this, it is possible to derive more specific rules such as 'adding or subtracting the same number to both sides of the equation' or 'multiplying or dividing both sides of the equation by the same number'. In the interviews, no student mentioned the general principle. Instead they focused on what they actually did as they solved the problems, which was described in terms of specific techniques such as 'pick up a number and put it to the other side of the equals sign changing its sign' or 'pick up a number and put it to the other side of the equals sign underneath whatever is in there.' Significantly, not one student explicitly articulated any understanding of mathematical reasons as to why these movements are appropriate actions to perform. It appears from their own description of what they were doing that they were simply moving symbols around. Symbols seem to be physical entities to them, that they can pick up and put where they feel appropriate. Essentially, the students were using their own ways of working with algebraic principles that were often misapplied as 'mal-rules' in the sense of Sleeman (1984). The dominance of specific techniques they had met before rather than the use of the general principles led to wide variety of errors that may seem almost random to an observer yet are an indication of the fragility of their knowledge.

We theorise that students remain with their human embodiment to shift the terms around to perform a sequence of operations in order to find the value of $x$. Using these techniques there appears to be no explicit difference in difficulty between the first two equations so the didactic cut was not statistically apparent.

However, the equation $2 m=4 m$, with the unknown occurring on both sides, does prove more difficult, with only seven out of 68 students giving the correct answer. As the unknown being on both sides was not a problem in the second equation, the difficulty in this case is likely to have a different source. Freitas (2002) claims that students have difficulties with multiplication and division involving zero. However, there may also be a problem with the embodiment itself. If $m$ stands for a physical object like a melon it does not make sense that two melons are equal to four melons (unless some are big and some are small!). On the other hand, if $m$ stands for something physical, how can it be zero and not be there? 
On studying all the data we found that none of the students mentioned that substituting the value $m=0$ makes the statement $2 m=4 m$ true. There is no evidence that the students are looking for a value of the unknown that satisfies the given equation. Instead there is a general tendency to shift the symbols around to move towards the desired solution.

Typical mistakes reported by Freitas (2002) happened in our study. For instance, a student might begin the solution of the first equation with the following sequence:

$$
\begin{gathered}
3 x-1=3+x \\
3 x-x=3+1 \\
2 x=4,
\end{gathered}
$$

and then different students had various final steps including:
(1) $x=4-2$
(2) $x=\frac{4}{-2}$
(3) $x=\frac{2}{4}$

The initial steps represent the 'change sides, change sign' technique, while the final step in each case reveals a 'mal-rule' applying a specific rule with some erroneous variation: (1) passes the 2 over the other side and changes the sign, (2) correctly 'shifts the 2 over and puts it underneath' but also 'changes the sign', (3) shifts the 2 over and puts the 4 underneath. In all cases, the errors can have arisen from misapplied met-befores.

While there were few instances of these particular errors in this question-one making error (1), one error (2) and two error (3) - similar errors occur in other questions, with eleven cases of error (1) in total, four error (2), and fourteen error (3). Allowing for two students making different errors on different questions, 27 out of 68 students made such errors.

\subsection{Students' own ways of working through procedural embodiment}

Some students become very attached to the use of techniques demonstrated by teachers and their own ways of working (derived from their earlier met-befores). For instance, in interviews, students made comments such as 'put all that is $x$ on one side, what is not to the other,' or 'when [the term] changes its place, [it] changes sign as well.' Another said, 'As it was multiplying on one side, it passes to the other dividing.' When asked why, a common explanation was that 'it is because that is the way to solve equations', or 'because the teacher said so'; none said anything about general algebraic principles or maintaining equality. There was no evidence in the responses that the students exhibited any understanding of the underlying mathematical methods.

In the following written solution: 


$$
\begin{aligned}
3 x-1 & =3+x \\
3 x-3 x & =+1 \\
0 & =\frac{1}{0}=0
\end{aligned}
$$

the student has shifted over the -1 on the left to give +1 on the right, but the $3+x$ on the right has become $-3 x$ on the left. The step from the second line to the third is a combination of replacing $3 x-3 x$ by 0 , then the ' $=+1$ ' is written as ' $=1$ ', and ' 0 ' is written below, (perhaps the 0 on the left has been moved over and below), and the line is completed by writing an 'answer' equal to zero (reflecting the difficulties students have with zero noted by Freitas (2002)).

All seven successful solutions to the equation $2 m=4 m$ involve a combination of symbol-shifting, changing sign and position, and some arithmetic simplification. A typical written solution, with verbal comments by the student, is:

$$
\begin{array}{rlrl}
2 m & =4 m \\
\text { "pass" } 4 m \text { to the other side: } 2 m-4 m & =0 \\
\text { add like terms: } & \text { "pass"-2 to the other side, dividing: } & m=0 \\
\text { to give the solution: } & m=0 .
\end{array}
$$

One student with written satisfactory solutions to the first two equations, wrote down the first three steps as above, but then wrote

$$
\begin{aligned}
-2 m & =0 \\
m & =\frac{-2}{0}=-2 .
\end{aligned}
$$

In the final line, there is a mal-rule (writing the quotient upside down) and a problem with dividing by zero, which is dealt with by omission, leaving an 'answer' -2 .

Another met-before that influences students' work is the rule saying, 'multiply a negative number by -1 to get a positive number'. Three students made the step of multiplying an individual term by -1 without regard to the meaning within the equation as a whole, as in the following example (which was placed in the category "Other solutions" in Table 1): 


$$
\begin{aligned}
2 m & =4 m \\
2 m-4 m & =0 \\
-2 m & =0 \\
m & =\frac{0}{-2} \\
m & =-0 \\
m & =-0 .(-1) \text { (multiplying one term by }-1) \\
m & =0 .
\end{aligned}
$$

Some students develop ways of working using a method that they are confident is true without needing to reason about it. However, trusting in such a procedure does not necessarily make it easy to use consistently.

\subsection{Met-befores from arithmetic and algebra}

Arithmetic has played a dominant role in previous learning and experiences from arithmetic may act as met-befores in various contexts. For instance, an individual who knows $3+2$ is 5 and does not know what to do with the $x$ in $3+2 x$ may perform the arithmetic operation and leave the $x$ alone to get $5 x$. Attempting to use experiences from arithmetic in algebra can leave to all kinds of mal-rules as in the following solution.

$$
\begin{aligned}
3 x-1 & =3+x \\
3 x & =-1+3 x \\
3 x & =-2 x \\
& =3 x-2 x \\
& =1 x
\end{aligned}
$$

From the first line, the term -1 is shifted to the other side and the $3+x$ on the right seems to be combined to give $3 x$. Then $-1+3 x$ is simplified to $-2 x$ (perhaps adding the numbers and retaining the minus sign). The terms $3 x$ and $-2 x$ are now shifted together and combined to give an 'answer' as $1 x$.

The most common student response seems to involve doing operations to the symbols to produce a solution. In a student interview an equation was described as 'many calculations in only one' and the solution is sought by shifting the terms around using given rules. The idea of searching for numbers to make a mathematical statement true does not seem to occur, nor does the idea of a balance between the two sides that must be maintained by operating on both sides in the same way.

Met-befores associated with algebraic expressions also influenced the students' work including ways of working with 'letters' using rules such as 'the rule that says that you cannot put together an unknown with another number that does not have the unknown'. These rules prevent students from adding unlike terms in equations, but 
fail to give any meaning to the expressions. Overall, formulating ways of working with algebraic expressions and equations did not seem to help most of the students to give a mathematical meaning to the symbols.

\subsection{A met-after from algebra}

The quadratic formula also influenced at least two students in their solutions of linear equations. For example, to solve the equation $5 t-3=8$, one student took $a=5$, $b=-3$ and $c=8$, and used the formula $\frac{-b \pm \sqrt{b^{2}-4 a c}}{2 a}$ to find a value for $t$.

At least three other students interpreted the equals sign as multiplication, for instance, one student wrote:

$$
\begin{gathered}
3 x-1=3+x \\
9 x+3 x^{2}+3+x= \\
10 x+3 x^{2}+3
\end{gathered}
$$

These students had studied quadratic equations in the previous academic year, where the focus was mainly on the use of the quadratic formula (Lima \& Tall, 2006). Now they and now seem to be searching for a way to use the quadratic formula to solve these equations. We therefore come to our first 'met-after' in which the students' thinking in solving linear equations is changed by their later experience with quadratic equations.

Met-afters, like met-befores, can have both positive and negative consequences. In the case above, the example shows a negative met-after, in which current knowledge is misapplied in a solving an earlier problem. For a met-after to have negative consequences like this, the earlier learning is likely to be fragile to be affected in this way. Identifying met-afters (as well as met-befores) could help the teacher to know what needs re-addressing in their teaching in new situations that not only apply to current learning but also to the stages before.

\section{DISCUSSION}

\subsection{Implications from the data}

In considering students' difficulties in solving linear equations, we have found much empirical evidence that the students in this study 'shift the symbols around' following their natural human inclinations to embody their actions with physical meaning. While their previous experience in whole number arithmetic allowed them to embody the arithmetic operations in terms of human actions on perceived objects, the shift to algebra is not so easily embodied. The idea of an equation as a balance works well for simple cases like $3 x+2=8+x$ but it fails when subtraction or negative numbers are involved. This leads to our first observation: 
1. While whole number arithmetic is readily embodied by seeing the symbolism in terms of actions on physical objects, the shift to algebra does not have an easy embodiment that covers all cases.

On the other hand, the students in this study do not build on algebraic expressions as processes. They do not see an equation such as $5 t-3=8$ as a process that can be 'undone' to find the value of $t$ that makes the equation true. In this case, theories of process-object encapsulation (Dubinsky, 1991, Sfard, 1991, Gray \& Tall, 1994) are not relevant to describe what the students are actually doing:

2.The students in our sample do not encapsulate algebraic processes as objects.

Nor do they build explicitly on general principles:

3. These students do not use the general principle of 'doing the same thing to both sides' as a basis for building up methods of solving equations.

Formal ideas such as this come much later in cognitive development, when the learner has experiences upon which these more formal methods can be built. While such an approach evidently appeals to experts who may have access to more formal thinking, the use of general principles is less meaningful for inexperienced learners. In our study the students focus not on the general principle but on the specific principles that they use in practice, such as 'picking up a term' and 'shifting it to the other side', while adding an additional piece of magic to 'change the sign'. While some more successful students clearly work in a meaningful way, others produce successful written solutions using their procedural embodiments accurately without understanding why they work.

4. Many students shift the symbols around to determine the answer using personal ways of working with symbol manipulation.

As observed by Payne and Squibb (1990), the less successful students have a wide array of mal-rules based on their experience of how they have been taught and what they actually do. The data shows:

5. These students operate functionally in an embodied manner, mentally moving symbols around.

Lakoff (1987, pp. 12-13) distinguished between conceptual embodiment and functional embodiment in a way that is helpful here: we interpret the procedural embodiment exhibited in the movement of algebraic symbols as a form of functional embodiment that may be performed without meaning.

Overall, our view of the data shows algebraic symbolism being underpinned by human embodiment and symbolic manipulation, leading to the overall conclusion:

6. A theoretical framework is needed to complement human embodiment and mathematical symbolism and link them together in the long-term development leading eventually to formal mathematical theory. 
Such a theoretical framework is formulated in the theory of three worlds of mathematics (Tall, 2004a, 2004b). In elementary mathematics, embodiment and symbolism grow in parallel, with each having meanings in different ways. In formal mathematics, interesting properties experienced through embodiment and symbolism in school mathematics are used as the basis for a third world of formal-axiomatic definition and mathematical proof.

\subsection{Teaching implications}

Some students find algebra simple and such students have learnt algebra over the years by a range of different approaches. However, other students seem to find it incredibly confusing, producing fear, anxiety and even loathing of the subject. Is it necessary to subject them to an experience which makes no sense to them, or are there ways in which algebra can be taught meaningfully?

Our hope is that we can develop a more realistic and meaningful approach to mathematics in general and algebra in particular with an understanding of who can benefit from its learning and how approaches to the subject can be organized to give successful experiences to those being taught. This does not necessarily entail teaching 'algebra for all' or developing approaches that have a short-term success without a long-term development.

We suggest that there is a need to develop a framework that is spoken easily by teachers and students as well as researchers. Learning builds on what we have experienced before. Our met-befores are the building blocks that we use as a base for our learning. Therefore, when misconceptions occur, it may be helpful to get students and teachers to talk about how ideas they have met-before ${ }^{2}$ affect our current learning

The term 'embodied' is used with a range of different meanings. Lakoff and Nunez (2000) see everything as embodiment. In our experience, many teachers find the meaning of 'embodiment' difficult to pin down. In translating ideas into other languages, such as Spanish, Greek and Chinese, translators have had difficulty with the term. It is therefore important to convey a suitable meaning for 'conceptual embodiment' in simple terms. In essence it involves putting together things that teachers already find familiar: coupling perception (mainly through visualization) with the results of our personal dynamic actions to build thought experiments to reflect on what happens in given situations.

A great deal of mathematical teaching currently involves the practice in carrying out specific algorithms: addition, subtraction, multiplication, division, using decimal notation, factorizing expressions, solving equations, using the rules of differential

\footnotetext{
${ }^{2}$ The term 'met-before' began as a joke, to contrast the practical 'met-B-fore' with the philosophical met-A-phor, and it works well in English. The evocative use of the term contrasts nicely with the notion of 'met-after'. In other languages one needs a friendly term to refer to things that are familiar from previous activity, for instance, in Portuguese the term 'já-encontrado' means 'already met' and 'a-encontrar' means 'to be met' and these terms are easily used in discussion with non-experts.
} 
calculus, and so on. This is a valuable experience in routinizing actions so that they can be carried out automatically. Our data shows that we should beware that such a practice can lead to a fragile form of procedural embodiment, shifting symbols without meaning. We know that there are widespread difficulties in the learning of a range of new ideas: in handling fractions, negatives, algebraic expressions, and so on. In all these areas an expert shifts the focus from the steps of the procedures concerned to the overall effect of that procedure to compress knowledge and make it more easy to manipulate in a sophisticated way. Many children, however, remain with the comfort of learned procedures and progress less easily to the more flexible use of symbols as process and concept, a phenomenon which Gray and Tall (1994) called 'the proceptual divide'.

In carrying out procedures, such as factorizing the expression $x^{2}-4$ into $(x-2)(x+2)$, the focus is initially on the steps that are performed to change one expression into the other. Another procedure of multiplying out expressions allows the second to be translated back to the first. However, the effort in translating one form into another separates them in time, and may maintain the perception that they are different. In the curriculum the equation $x^{2}-4=(x-2)(x+2)$ is regarded as a special case of an equation: it is an identity because it is true for all $x$. Subsequently, the functions $f(x)=x^{2}-4$ and $g(x)=(x-2)(x+2)$ are declared to be precisely the same function. In this succession of conceptions, we see a shift from distinct procedures to the same input-output process giving the same function concept. Yet this shift from procedure to process to thinkable concept is not usually made explicit, nor does it seem to be easy. We believe that a focus on the effect of an action can help to make this shift in conception more evident. Two functions given by different formulae are the same precisely when they have the same effect.

By using two distinct ways of introducing a new concept through embodiment and symbolism, it is possible to see symbolic process-object compression having a parallel construction in the embodied world. Starting from physical procedures that embody the symbolic procedures, then looking at different procedures that have the same effect, gives a parallel embodied development to the first part of the theoretical APOS construction from Action to Process. If that process can be embodied in a physical way, then this enables the further shift from embodied process to embodied object, as Watson (2002) showed in the case of a vector, shifting from a translation of an object, to the effect of the translation as a shift, to the representation of that shift as a free vector embodied as an arrow of given magnitude and direction that can be placed anywhere.

The same shifts of attention work in other contexts. The effect of the action of counting a collection is the last number word spoken. In this way there is a shift in attention from various procedures of counting to the concept of number. Fractions are procedures of sharing and the goal is the quantity produced by the procedure. Equivalence of fractions is more easily seen in terms of different sharing procedures 
having the same effect. Equivalence of algebraic expressions occurs when generalized arithmetic expressions have the same effect when evaluated for all specific numerical values of the variables. We believe that the notion of 'same effect' can be used to underpin the notion of 'equivalence' of operations leading over time to helpful meaning for the mathematical notion of equivalence.

In calculus, the subtle notion of derivative has an embodied meaning as the steepness of the graph itself. By magnifying the graph (of a differentiable function) highly until it looks straight in a computer window or in the mind's eye it is possible to 'look along the curve' and experience the changing slope as a function. With a computer, it is possible to guess the formula for each of the standard derivatives before the symbolic limit process need be introduced to confirm it. The formal language of 'existence' is irrelevant when the embodied concept can be seen. The symbolic limit concept is now a subtle way of computing the derivative as a symbolic expression. Once more, the embodied world gives a meaning for the derivative function as the graph of the changing slope before the symbolic limit is introduced to calculate it.

\subsection{Some suggestions}

The previous discussion provides evidence that a theoretical framework that balances the development of embodiment and symbolism can assist in curriculum design. It is not enough to use embodiment in terms of a physical 'balance' that works well in simple cases but does not generalize. It is not sufficient to build symbolic ideas on undoing evaluation equations (of the type 'expression equals number'). Nor is it appropriate to build from higher level principles of 'doing the same thing to both sides' if this defaults to the procedural embodiment shifting symbols with added 'magic' of 'change sides, change signs' or 'move it over and put it underneath'.

It is far more important to build on what learners experience and what they do. This needs a teacher who is sensitive to the students' current knowledge and has experience in helping them make sense of new ideas. It may involve revisiting earlier situations at appropriate times to recognize explicit met-befores that continue to work in those situations, but are no longer appropriate in the new context. It may involve recognizing earlier fragility of knowledge exhibited by met-afters in subsequent knowledge that causes a rethink of how to teach the earlier knowledge in future.

In the long-term learning of increasingly sophisticated mathematics, it is important for the teacher to be aware of the principle of compression of knowledge into thinkable concepts by focusing on relevant aspects. In particular it is essential to consider how to shift attention from the actions performed to the more important effect of those actions. We hypothesize that such insights can support the student in the long-term development moving through the increasing sophistication of arithmetic, algebra, the function concept and its later developments in calculus and the use of these tools in solving novel mathematical problems.

The need for compression of knowledge highlights the distinction between procedural and conceptual knowledge. The term 'conceptual' relates to making 
connections in knowledge networks. For connections to be possible, the nodes of the networks need to be thinkable concepts, the connections need to be made not only through giving embodied meaning to symbolic concepts, but also to make sense within the symbolic world itself. Compression occurs by considering several different procedures with the same effect as a single process that may be carried out in several different ways. By linking symbolism and embodiment, a process may be embodied as a mental object before it is encapsulated in a symbolic sense. The shift from procedural to conceptual knowledge then involves not only embodied meaning of symbolism, but also coherent links within the symbolism itself.

\section{References}

Anderson, C.: 1997, Persistent errors in indices: a cognitive perspective. PhD Thesis University of New England, Australia.

Brasil. Secretaria de Educação Fundamental: 1998, Parâmetros Curriculares Nacionais: Matemática. Brasília: MEC/SEF.

Brousseau, G.: 1997, Theory of Didactical Situations in Mathematics. Kluwer, Netherlands.

Cortés, A., Pfaff, N.: 2000, Solving equations and inequations: operational invariants and methods constructed by students. Proceedings of the $24^{\text {th }}$ Conference of the International Group for the Psychology of Mathematics Education, Hiroshima, Japan, vol. 2, p. 193200.

Dubinsky, E.: 1991, Reflective abstraction in advanced mathematical thinking. In D. O. Tall (Ed.), Advanced Mathematical Thinking, (pp. 95-123). Dordrecht: Kluwer.

Filloy, E. and Rojano, T.: 1989, Solving equations, the transition from arithmetic to algebra, For the Learning of Mathematics, 9(2), 19-25.

Freitas, M. A. de: 2002, Equação do primeiro grau: métodos de resolução e análise de erros no ensino médio. Master's Dissertation. São Paulo: PUC-SP.

Gray, E. \& Tall, D. O.: 1994, Duality, Ambiguity and Flexibility: A Proceptual View of Simple Arithmetic, The Journal for Research in Mathematics Education, 26(2), 115-141.

Hart, K. M., Johnson, D. C. (ed), Brown M., Dickson L., Clarkson, R.: 1989, Children's Mathematical Frameworks 8-13: A Study of Classroom Teaching, Routledge (formerly NFER Nelson).

Kieran, C.: 1981, Concepts associated with the equality symbol, Educational Studies in Mathematics, 12, 317-326.

Lakoff, G.: 1987, Women, Fire and Dangerous Things, Chicago, IL: University of Chicago Press.

Lakoff, G. \& Nunez, R.: 2000, Where Mathematics Comes From. New York: Basic Books.

Lima, R. N. \& Tall, D. O.: 2006, The concept of equations: What have students met before? Proceedings of the $30^{\text {th }}$ Conference of the International Group for the Psychology of Mathematics Education, Prague, Czech Republic, vol. 4, 233-241.

Payne, S. J. \& Squibb, H. R.: 1990, Algebra Mal-Rules and Cognitive Accounts of Error, Cognitive Science 14, 445-448. 
Sfard, A.: 1991, On the dual nature of mathematical conceptions: reflections on processes and objects as different sides of the same coin. Educational Studies in Mathematics. Kluwer Academic Publishers. The Netherlands. Vol. 22, p. 1-36.

Simon, M., Tzur, R., Heinz, K. \& Kinzel, M. (2004). Explicating a Mechanism for Conceptual Learning: Elaborating the Construct of Reflective Abstraction. Journal for Research in Mathematics Education, 35(1), 305-329.

Sleeman, D. H.: 1984, An attempt to understand students' understanding of basic algebra, Cognitive Science, 8, 387-412.

Tall, D. O.: 2004a, The three worlds of mathematics, For the Learning of Mathematics, 23(3). 29-33.

Tall, D. O.: 2004b, Thinking through three worlds of mathematics, Proceedings of the $28^{\text {th }}$ Conference of the International Group for the Psychology of Mathematics Education, Bergen, Norway, 4, 281-288.

Tall, D. O. \& Thomas, M. O. J.: 2001, The long-term cognitive development of symbolic algebra, International Congress of Mathematical Instruction (ICMI) Working Group Proceedings - The Future of the Teaching and Learning of Algebra, Melbourne, 2, 590597.

Tirosh, D., Even, R. \& Robinson, N. (1998), Simplifying algebraic expressions: teacher awareness and teaching approaches, Educational Studies in Mathematics, 35, 51-64.

Thurston, W. P.: 1990, Mathematical Education, Notices of the American Mathematical society, 37(7), 844-850.

Vlassis, J.: 2002, The balance model: hindrance or support for the solving of linear equations with one unknown, Educational Studies in Mathematics. Kluwer Academic Publishers. The Netherlands. Vol. 49, p.341-359.

Watson, A.: 2002, Embodied action, effect, and symbol in mathematical growth. Proceedings of the $26^{\text {th }}$ Conference of the International Group for the Psychology of Mathematics Education, Norwich, UK, 4, 369-376.

Watson, A., Spyrou, P., Tall, D. O.: 2003, The Relationship between Physical Embodiment and Mathematical Symbolism: The Concept of Vector. The Mediterranean Journal of Mathematics Education. 1(2), 73-97. 